\title{
The Application of Magnetometers and Electromagnetic Induction Sensors in UXO Detection
}

\author{
Songtong Han ${ }^{1}$, Xiaoli Rong ${ }^{1, ~}{ }^{*}$, Leixiang Bian ${ }^{1}$, Mingyou Zhong ${ }^{1}$, and Lining Zhang ${ }^{2}$ \\ ${ }^{1}$ School of Mechanical Engineering, Nanjing University of Science and Technology, Nanjing 210094, China \\ ${ }^{2}$ School of Life Science, Beijing Institute of Technology, Beijing 100081, China
}

\begin{abstract}
This paper introduces the physical model of unexploded ordnance (UXO) in the magnetization process and the formula derivation of the magnetic dipole model. The application of magnetometers and electromagnetic induction sensors in the detection of UXO is introduced. The magnetometers introduce the total field measurements technology of the CS optical-pump marine magnetometer and the MagSTAR (Magnetic Scalar Triangulation and Ranging) gradient detection technology. The electromagnetic induction sensors introduce the working theories and the popular product models of Geophex company and Geonics Ltd. The method of detecting UXO by MTADS of United States Naval Research Laboratory (The multisensor towed array detection system) compares the difference between the United States Naval Research Laboratory for target recognition.
\end{abstract}

\section{Introduction}

Unexploded ordnance (UXO) mainly refers to the fault bombs left over from the war and the mines left by the minefield [1]. According to the International Campaign to Ban Landmines (ICBL), more than 80 countries and regions in the world are affected by landmines and UXOs to varying degrees. From 1999 to 2005, more than 42,500 people in the world were injured by landmines and UXO [2]. In 2015 alone, the number of people killed and killed in booby traps due to accidental treading reached 6,461. According to reports, from the 1970s to the 1990s, about 1.3 million landmines and more than 480,000 kinds of explosives were planted in the Yunnan section of the Sino-Vietnamese border, forming 161 chaotic minefields with an area of about 289 square kilometers. Currently, the Sino-Vietnamese border in Yunnan. The area of the minefield is about 76.3 square kilometers, and there are about 157,000 explosives such as unexploded grenades and shells. These UXO seriously affect the normal life of local villagers.

China's detection of underground UXO started late, and there is still a big gap compared with the mature technology in the West. This paper mainly introduces the basic model of UXO and two methods commonly used for UXO detection (Magnetic and Electromagnetic methods), I hope to help relevant workers in foreign countries.

\section{UXO's physical models}

When a magnetic field is present, the substance is magnetized, that is, the tiny dipoles contained in the substance tend to align in a certain direction. This trend can be divided into two mechanisms:

- Paramagnetic (the dipole associated with unpaired electron spins is subjected to a moment that causes them to align in a direction parallel to the direction of the outer field),

- Diamagnetic (the orbital velocity of the electron is Change, this reason causes the orbital magnetic moment to produce a variable opposite to the direction of the external field) [3].

The degree of magnetization can be described by equation (1)

$$
\mathbf{M}=\frac{\sum \mathbf{m}_{\mathrm{mo}}}{\Delta v} .
$$

Where $\mathbf{m}_{\mathrm{mo}}$ is the molecular magnetic moment and $\mathbf{M}$ is the magnetization vector (unit $\mathrm{A} / \mathrm{m}$ ), which is the vector sum of the molecular magnetic moments per unit volume.

The magnetization process can be understood as the formation of magnetizing current on the outer surface of the magnetized medium (the ampere molecular current point of view, we will not discuss the magnetic charge point here). It can be seen from the equation (2) that the surface magnetization current $\mathbf{i}$ is equal to the cross-over of the magnetization vector $\mathbf{M}$ and the unit vector $\mathbf{n}$ of the magnetized medium surface

$$
\mathbf{i}=\mathbf{M} \times \mathbf{n} .
$$

According to the direction of the magnetization current $\mathbf{i}$, the magnetization field generated by the magnetization medium after magnetization can be determined, but since there are many unknowns (elastic 
shape, azimuth angle, etc.), the model can be considered simplified. When the target detection distance is greater than 2.5 times the magnetic target length, the magnetization target can be regarded as a magnetic dipole. Under this condition, the magnetic anomaly field $\mathbf{B}(r, \mathbf{m})$ (unit $\mathrm{N} / \mathrm{A} \cdot \mathrm{m}$ ) from the magnetization target $\mathrm{r}$ can be obtained by the equation (3)

$$
\mathbf{B}(r, \mathbf{m})=\frac{\mu}{4 \pi}\left[\frac{3(\mathbf{m} \cdot \mathbf{r}) \mathbf{r}}{r^{5}}-\frac{\mathbf{m}}{r^{3}}\right] .
$$

Where $r$ is the target position vector, $\mathrm{m}$ is the magnetic moment of the magnetic target (unit $\mathrm{Am}^{2}$ ), $\mathrm{r}$ is the scalar of the magnetic target to the measuring point, where $r=|\mathbf{r}|, \mu$ is the environmental medium permeability, for non-magnetic targets the air environment is approximately $\mu=4 \pi \times 10^{-7}$ (unit $\mathrm{T} \cdot \mathrm{m} / \mathrm{A}$ ).

\section{Magnetic detection}

In geophysical exploration, magnetic detection is one of the earliest and widely used geophysical exploration methods, which has the following three distinctive features.

- Light and easy, high efficiency,

- The field of work is wide and not subject to geographical restrictions,

- A wide range of applications.

In modern times, due to the development of modern magnetism, the geomagnetic self-excited motor hypothesis [4] is proposed. The UXO is magnetized under the action of the earth's magnetic field, and the resulting magnetized field causes the original uniform local geomagnetic field to produce anomalies. Abnormal extraction analysis, and finally determine the location information of UXO.

Generally, the UXO uses metallic iron as the outer casing, and the metallic iron has ferromagnetism (which belongs to paramagnetic pro-perties with high magnetic susceptibility), and the larger the magnetization field, the more obvious the magnetic anomaly. Such anomalies can be determined by the content of ferromagnetic material, the thickness of the shell, the ratio of the length of the shell to its radius, the permeability and the direction of the geomagnetic field [5].

\subsection{Common magnetic sensor classification}

At present, magnetic sensors commonly used in magnetic detection mainly include optical pump magnetometers, proton magnetometers, fluxgate sensors, and superconducting quantum interferometers. The optical pump is a new technology developed in the 1950s. The optical pump magnetometer is a highsensitivity and high-precision magnetic measuring instrument. It is based on the Zeeman effect of the atomic energy level of the working substance in the magnetic field, plus optical pump technology and magnetic resonance technology.

The optical pump magnetometer has the Cs-3 neon pump magnetometer produced by Scitrex and the G-858 calender pump magnetometer from Gcometrice. Their sensitivity is $0.6 \mathrm{pT}$ and $0.01 \mathrm{pT}$ respectively, which basically represents the foreign beauty. The development level of optical pump magnetometers. In addition, it is reported that the United States Polato company replaced the original xenon lamp with a krypton laser, and developed a P-2000 He-light pump magnetometer with a much higher accuracy than the original He-light pump magnetometer, the sensitivity reached $0.3 \mathrm{pT}$, representing the current The development level of the world's Dawn Pump magnetometer [6].

The Proton Magnetometer is an absolute magnetometer for measuring the total strength of the Earth's magnetic field. It was developed by Packard and Varian in 1954 and is one of the most widely used geomagnetic survey instruments. The structure is simple, reliable, and inexpensive. However, the power consumption is large, and only intermittent measurement can be performed. The sensitivity is not high enough, and it is not suitable to be a gradiometer [7].

The fluxgate sensor is a device that measures the magnetic field by the nonlinearity in the saturation region when the ferromagnet is magnetized. When the ferromagnetic material made of soft magnetic material is magnetized, the external magnetic field can be modulated due to the nonlinearity of the magnetization, so that the sensor output Associated with an external magnetic field. Typical fluxgate sensors in the world include the Canadian FM200 and CANMOS fluxgate sensors, the Austrian CHIMAG fluxgate sensor, the UK FLARE fluxgate sensor, and the US SMALL fluxgate sensor [8].

The Superconducting QuantumInterference Devices (SQUID) was developed in the mid-1960s. It is based on the Josephson effect and is made of superconducting materials. Its sensitivity is theoretically up to $10^{-15} \mathrm{~T}$, which is the most sensitive magnetic field detection device known to man, and its measurement range is wide, from zero field measurement to thousands of Tesla, and the response frequency can range from DC to several gigabits. hertz. The best high temperature SQUID magnetic field sensitivity is currently 10fT/(Hz@1 $1 \mathrm{~Hz})^{1 / 2}$. But the magnetometer is too bulky [9].

\subsection{Total field measurement}

According to different methods of magnetic detection, it can be divided into total field measurement method and gradient measurement method. The total field measurement needs to be corrected by daily variation. Usually, the daily variation correction method is to install a same type of base station type magnetic sensor in a relatively stable magnetic environment for daily change observation and correction.

Fig 1 uses the G-858 pump to magnetically detect a $5 \mathrm{~m} \times 5 \mathrm{~m}$ area, and another G-858 pump to measure the daily variation. The UXO uses a standard $40 \mathrm{~L}$ industrial oxygen cylinder, and the north-south square orientation and distance plane $1.8 \mathrm{~m}$, the experimental location is somewhere in Nanjing. The blue point in the figure is the centroid position of the UXO. The right side of the 
figure is the geographical south side. Since the experimental position is in the northern hemisphere, the abnormal distribution can be clearly distinguished as the south-north-negative feature (the southern hemisphere concludes the opposite).

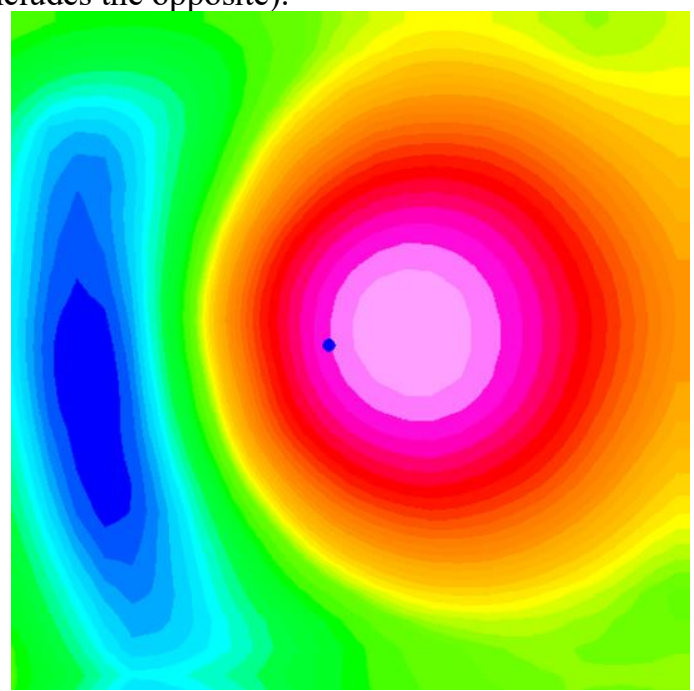

Fig. 1. Total magnetic field anomaly.

\subsection{Gradient measurement}

The gradient measurement uses a plurality of vector magnetic sensors to perform gradient measurement on each magnetic field component of the magnetic target, and does not require daily variation correction compared to the total field measurement.

The theoretical basis of the gradient measurement is to obtain the position information in the generalized coordinates by analyzing the magnetic vector potential generated by the magnetization target (as shown in Fig. 2). Taking the Cartesian coordinate as an example, the relative coordinates of $\mathrm{x}, \mathrm{y}$, and $\mathrm{z}$ of the magnetic dipole model are obtained. The magnitude of the magnetic vector potential can be derived from equation (4)

$$
\mathbf{G}=\nabla \mathbf{B} .
$$

Where $G$ represents the magnetic gradient tensor. The above formula can be reduced to a scalar form equation (5)

$$
\begin{aligned}
\mathrm{G}_{\mathrm{ij}} & =(\nabla \mathrm{B})_{\mathrm{ij}}=\frac{\partial \mathrm{B}_{\mathrm{i}}}{\partial r_{\mathrm{j}}} \\
& =\frac{-3 \mu}{4 \pi r^{-7}}\left[\mathbf{M} \cdot \mathbf{r}\left(5 r_{\mathrm{i}} r_{\mathrm{j}}-r^{2} \delta_{\mathrm{ij}}\right)-r^{2}\left(r_{\mathrm{i}} \mathrm{M}_{\mathrm{j}}+\mathrm{M}_{\mathrm{i}} r_{\mathrm{j}}\right)\right] .
\end{aligned}
$$

Where $\mathrm{i}$ and $\mathrm{j}$ represent the Cartesian coordinate system parameter components, $\delta_{\mathrm{ij}}$ is the Kronecker symbol, when $\mathrm{i}=\mathrm{j}, \delta_{\mathrm{ij}}=1$, if $\mathrm{i} \neq \mathrm{j}, \delta_{\mathrm{ij}}=0$.

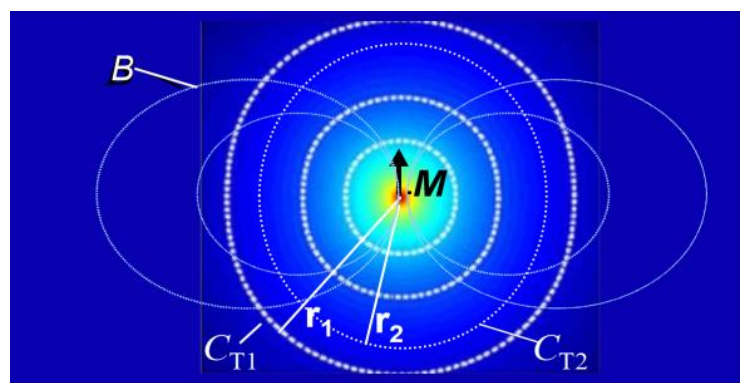

Fig. 2. Magnetic induction intensity and magnetic vector potential distribution of magnetic dipole model.

The MagSTAR (Magnetic Scalar Triangulation and Ranging) system developed by the US Navy uses gradient measurement technology. The system uses eight low-noise $\quad\left(20 \mathrm{pT} /(\mathrm{Hz} @ 1 \mathrm{~Hz})^{1 / 2}\right)$ three-axis fluxgate sensors to form a gradient magnetic detector (as shown Fig 3) with a detection range of $\pm 5000 \mathrm{nT}$ and a sensitivity of $0.02 \mathrm{nT} / \mathrm{m}$ for magnetic moments. The target of $14.5 \mathrm{Am}^{2}$ can detect the arrival distance of more than $5 \mathrm{~m}[10-13]$.

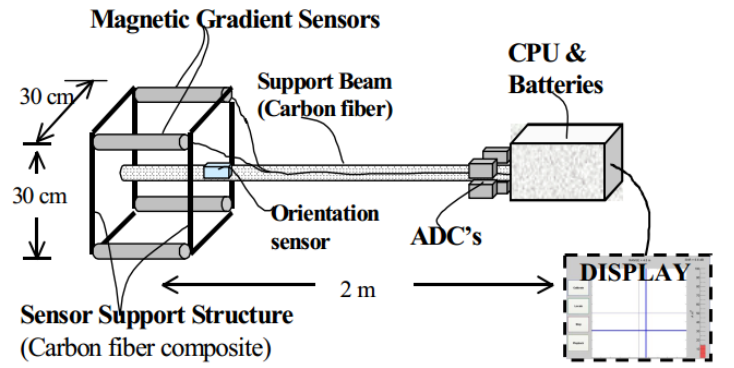

Fig. 3. Schematic diagram of MagSTAR system structure.

\section{Electromagnetic detection}

Electromagnetic detection is also called active magnetic detection. Its working principle is active coil excitation, generating magnetic field signals. The UXO is magnetized by the magnetic field generated by the active coil to generate a secondary field. At this time, the secondary field is detected. The position information of the unexploded bullet is shown in Fig 4. The analysis and processing of signals is generally based on two categories: time domain analysis (TDEMI) and frequency domain analysis (FDEMI).

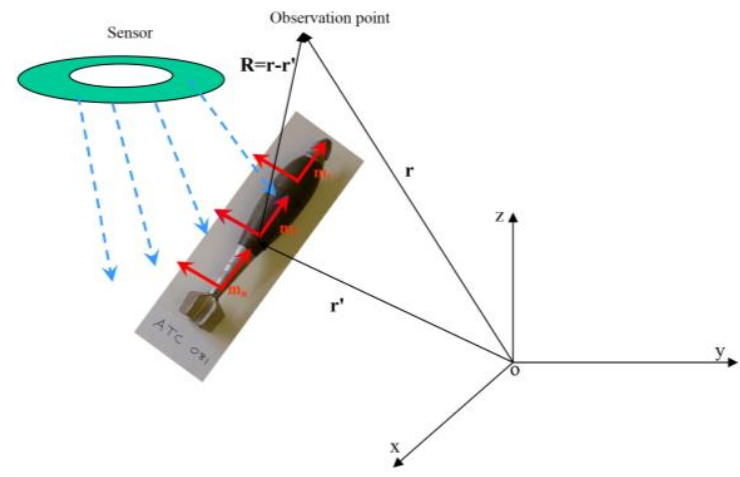

Fig. 4. Active magnetic detection schematic.

Electromagnetic detection of UXO is more prevalent in foreign countries, especially the classification of UXO and metal debris, as well as the identification of UXO, and even the United Nations weapons inspectors search for geophysical weapons of Iraq's weapons of mass 
destruction. The same is true for exploration workers. Although the equipment and working methods are more complex than the magnetic method, they first chose the electromagnetic method for detection [14-16].

At present, there are many UXO products based on electromagnetic method, and the technology is mature. For example, Geophex Co., Ltd. of Canada has been engaged in electromagnetic detection for 30 years. Products GEM-2, GEM-3, GEM-3R, GEM-3 Arry, GEM-5 Arry has a good performance in UXO detection (see Fig 5).

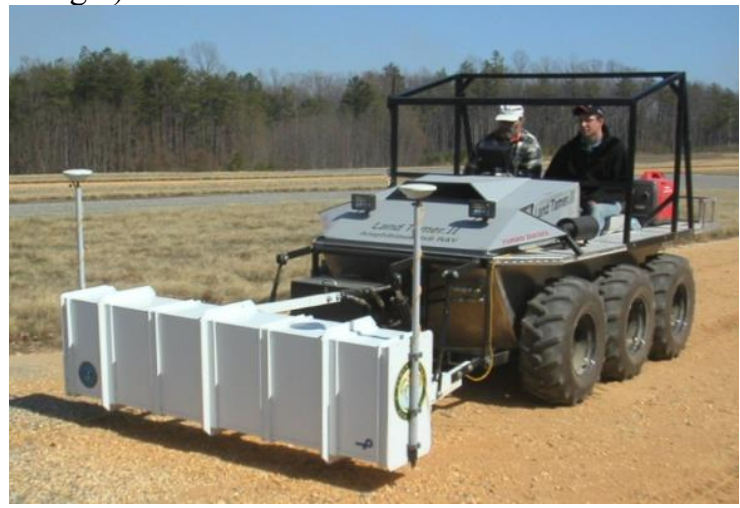

Fig. 5. GEM-5 Array.

Geonics's EM61-MK2A, EM61S, EM61HH-MK2A, EM63-3D-MK2 and other products have excellent performance in different fields, among which EM61MK2A is considered as the standard sensor technology in the environmental and military fields, EM61- The MK2A has a maximum depth of detection of 5.5 meters, depending on the target characteristics. The EM63-3DMK2 is considered the most advanced instrument for distinguishing and classifying UXO (as shown in Fig 6), and the EM63 is capable of providing complete transient attenuation changes for metal targets.

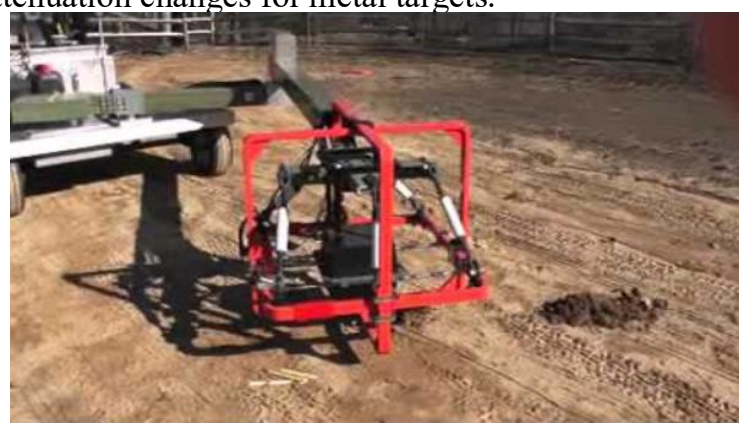

Fig. 6. EM63-3D-MK2.

\section{Conclusion and outlook}

The above two methods for detecting UXO, the magnetic detection system is simpler and more affordable than the electromagnetic detection system. If the sensor with higher resolution and sensitivity is used, the positioning accuracy is higher, but the magnetic field is stable to the surrounding environment. The performance (small disturbances and anomalies) is highly dependent; the electromagnetic detection system is more expensive and the system is more complex, but it can also perform well in complex environments, and at the same time classify and identify UXO.
As early as the end of the 1990s, the U.S. Naval Research Laboratory used magnetic and electromagnetic methods to make experimental comparisons of UXO [17-18]. The report pointed out that there are different advantages for the detection of UXO and electromagnetic methods. Some magnetic methods have not detected the UXO and the electromagnetic method has detected it. The same is true for UXO that cannot be identified by some electromagnetic methods. The magnetic method can be identified, and when the two methods have a common misjudgment, the detection and recognition rate of the two combined detection is as high as $95 \%$.

Every year, tens of thousands of civilians have lost their lives because of UXO. The author lives in the countryside of a city in the northeast. When he was a child, he witnessed the excavation of Japanese howitzers on the dirt roads of the village. Although the shell fuzes have failed, Every thought is so intense that I hope that civilians will no longer suffer from UXO.

I would like to use this document for workers who are engaged in the detection of UXO, and I hope that it will help.

\section{References}

1. W. Zhang, Y. Liu, X. Zhang, Chn. Geps. Soc (2012)

2. C. Chirgwin, Leading Edge, 24, 128-140 (2012)

3. S.L. Dell. R.K.P Zia. Princeton University Press, 4, 3 (1979)

4. F. Press, Siever R.Earth, 459-460 (1986)

5. D.K. Butler, The Lea ding Edge, 20, 890-895 (2001)

6. T.J. Gamey. W.E. Doll. L.P. Beard, J. Env \& Eng. Geps, 9, 115-125 (2004)

7. B. Qin, H. Pre. Mag. Exp, (1988)

8. N. Tu, W.S. Guo, D.P. Cao, Min. War. S. Pro, 1, 4042 (2002)

9. J.B. Lee, D.L. Dart, R.J. Turner, Geo, 67, 468 (2002)

10. R. Wiegert, J. Oeschger, Oceans IEEE, 2, 13251332 (2006)

11. R. Wiegert, K. Lee, J. Oeschger, Oceans IEEE, 1 (2008)

12. R. Wiegert, J. Oeschger, Oceans IEEE, 1 (2006)

13. R. Wiegert, Pro. SPIE (2009)

14. I.J. Won, V. Murphy, P. Hubbard, L. E, 23, 658-662 (2004)

15. Z. Qu, Y.T. Li, Geo. S. Tec. Inf, 25, 103-106 (2006)

16. Q.K. Meng, W. Gao, Y.C. Wang, N. S. Geo. S (2017)

17. H.H. Nelson, T.H. Bell, J.R. Mcdonald, A. M. C. Det \& Dis. U (2003)

18. H.H. Nelson, J.R. Mcdonald, IEEE. T. Geo \& Rem.S, 39, 1139-1145 (2001) 